\title{
INFRA-RED SPECTRA AND THE STRUCTURE OF MOLECULES*
}

\author{
By DR. W. H. J. Childs and DR. H. A. JAHN, \\ Royal Institution, LondoN.
}

$\mathrm{W}^{\mathrm{E}}$ $\mathrm{E}$ have to go back only to 1800 , to $\mathrm{W}$. Herschel's discovery that beyond the red end of the sun's prismatic spectrum there is something capable of warming the bulb of a thermometer, to find the germ of a method which, through its contributions to our knowledge of molecular structure, is beginning to be of much importance both to chemistry and to physics. Herschel's discovery was accompanied by an error-that the energy maximum of the spectrum lay also outside the red-though this probably made it even more effective in stimulating the curiosity and imagination of contemporary men of science. In spite of this interest, for the rest of the century progress was slow, and this period is taken up first with the need of proving that the new rays are nothing other than invisible light and obey exactly the same laws; and then with the task of mapping the new region, to find out if and how the radiation is absorbed by matter.

On looking back, we see that the real advances are the improvements in technique, mainly concerned with increasing the sensitivity of radiation detectors. Towering above all in its effect was the discovery by Rowland of a method of making a perfect screw, and with it constructing the concave diffraction grating. These technical advances have made their permanent impression, and we find to-day that the thermopile and the diffraction grating in their modern improved forms are playing a leading part in infra-red research. At the beginning of the twentieth century, though many absorption spectra in the region $1-20 \mu$ had been measured and it was known that every substance, with the exception of the diatomic homopolar molecules such as hydrogen and nitrogen had characteristic absorption bands in the infrared, no success had attended the few attempts empirically to systematize this knowledge. Indeed it is only in recent years, following upon the successful disentangling of the spectra of diatomic molecules with the help of quantum theory, that infra-red absorption bands are being related to other molecular magnitudes.

The starting point of the theoretical interpretation of infra-red molecular absorption bands is the Faraday-Maxwell electromagnetic theory of radiation. Once it was clear that radiation consists of electrical oscillations, the absorption of

-Substance of a course of seven lectures delivered at the Royal Institution between November 3 and December 15, 1939. infra-red radiation by molecules could be explained on the basis of resonance between the electrical oscillations of the individual molecules and the oscillations of the electromagnetic field. The occurrence of characteristic frequencies in the absorption spectrum pointed to the existence of a definite spectrum of frequencies possessed by, and characteristic of, each individual molecule, this again pointing to a definite mass distribution and force system in the molecule.

Before Rutherford's nuclear theory of the atom, however, very vague ideas were current about the distribution of mass in the molecule. Molecules were considered to be built up of spherical ions, and characteristic frequencies of the individual ions were often spoken of, as would be possible if there were a characteristic distribution of mass in the ion itself. With the advent of Rutherford's theory, the distinction was quickly made between electronic frequencies involving the electronic mass, and the much slower frequencies involving the nuclear masses. It is these latter frequencies which show up in the infra-red absorption bands. Thus, for example, the water molecule is to be regarded as consisting of three heavy mass points held together in their equilibrium positions by electrical forces. It is easy to see that such a mechanical system possesses three degrees of vibrational freedom, so that the water molecule should have (as is indeed the case) three different molecular frequencies showing up in its absorption spectrum. It was this clarification of the situation that enabled Bjerrum in 1914 to lay the foundations of the method of calculating molecular frequencies still in use to-day. It had been pointed out still earlier by Lord Rayleigh that if the electrical oscillations have definite fixed directions in the molecule, then the rotational frequencies of the molecule will also appear in the spectrum, since the carrying round of the fixed electrical oscillation by the molecule will be equivalent to a harmonic oscillation having the same period as the molecular rotation, which will thus appear combined with the vibrational frequencies in the spectrum.

For the purposes of review, we may conveniently separate infra-red spectrographs into two classes, low resolving power prism and high resolving power grating instruments. The prism spectrograph is adequate to measure band envelopes, but for the finer structure due to molecular 
rotation, and to separate overlapping bands in the $3 \mu$ region, and above all for work in the very long wave infra-red in the region $30-100 \mu$, a grating instrument is essential. Thus these two classes, very broadly speaking, give us respectively information about molecular forces and molecular sizes. The prism spectrograph has usually a theoretical resolving power of some few hundreds, but this is reduced when in use (the need of using slits wider than the optimum is one reason) by a factor of from four to eight. There have been advances in design in recent years. Careful placing of the entrance and exit mirrors of the prism, so that the errors introduced into the beam by one are partially rectified by the other, has resulted in much improved definition in the spectrum falling on the exit slit. There is a noticeable tendency to place the whole spectrograph in an evacuated chamber, thus avoiding the loss of energy in those regions of the spectrum where strong absorption by the water vapour and carbon dioxide of the atmosphere occurs. For the prism and windows we are no longer dependent on natural crystals of rocksalt, as large enough crystals can now be grown from the melt. Crystalline potassium bromide obtained in this way has extended the range of prism instruments to nearly $30 \mu$.

Subsidiary apparatus has also been improved. Thermopiles with only one or two elements are constructed to have a resistance and speed of response to match the galvanometers with which they are to be used. The moving-magnet galvanometer has gone quite out of favour, and is replaced by a less sensitive but far more stable movingcoil instrument, connected by some form of photoelectric or thermo-electric amplifier to a second more rugged galvanometer from which the readings are taken. In some instances where careful design and construction have almost entirely eliminated disturbances and drifts, it has proved worth while to instal an automatic recorder.

With grating instruments the resolving power is usually of the order of several thousands, and although in practice the necessity of using slits wider than the optimum again sets the limit, nevertheless powers of 10,000 at $3 \mu$, and 300 at $80 \mu$, have been attained. The predominant type of grating is the 'echelette', with a predetermined groove-form to diffract the bulk of the energy in one particular direction. This property is a most desirable one, for the energy available is usually so meagre that its dissipation into spectra of more than one order cannot be tolerated. If it is desired to work in more than one region, several gratings must be employed, those for the longer wave-lengths having correspondingly wider grooves and thus, for the same resolving power, greater dimensions. Excellent gratings as large as ten inches by twenty inches have been produced for work at the longest wave-lengths, around $80 \mu$. The suppression of other orders and the scattered radiation of the more abundant Shorter wave-lengths around $3 \mu$, which would so to speak 'fog' the thermopile, is achieved with judiciously selected filters, or by making use of selective reststrahlen reflections, or by a combination of both.

By using vacuum thermopiles, carefully screened conductors and evacuated spectrographs, disturbances and drifts are reduced to a minimum, but even so, automatic recording is not possible in the longer wave-length regions unless a special tuned amplifier is used. With this ingenious device the beam of infra-red radiation is interrupted periodically by a shutter so that the deflection of the primary galvanometer is also periodic. The amplifier is tuned to the same frequency and is thus insensitive to drifts and all disturbances of arbitrary period. The deflections of the secondary galvanometer may then be recorded photographically, since its zero remains steady.

It is possible to make measurements in the very short-wave infra-red, from the visible to $1 \cdot 2 \mu$, with ordinary diffraction gratings and the specially sensitized photographic plates which are now commercially obtainable. The resolving power may be high, of the order of 20,000 , but the relatively simple technique is unfortunately limited to a few types of bands of molecules containing hydrogen. In these cases alone is the absorption great enough to permit the use of absorption tubes of practical length.

The theoretical analysis of molecular infra-red absorption bands can be divided into two fairly distinct processes: the vibrational and the rotational analysis. This division follows quite closely the division of the experimental work into prism and grating spectroscopy respectively.

In the vibrational analysis we consider the molecule to be built up of point masses (the atomic nuclei) held together by elastic forces, and the major problem is to calculate the classical normal modes of vibration of such a system. Such a calculation necessitates a knowledge of the equilibrium configuration of the molecule and a knowledge of the elastic forces which are called into play when the atoms are displaced from their equilibrium positions. The geometrical configuration is usually fairly well established from chemical considerations or from the electron theory of valency, whilst the force system also in many cases goes hand in hand with the chemical formulæ. Thus a good part of the vibrational spectrum can be interpreted on the basis of 
characteristic stretching constants for the individual chemical bonds. A molecule possesses, however, in addition to such valency vibrations, deformation modes in which only the angles between chemical bonds change. It is tempting to introduce individual characteristic elastic constants for the angles between the bonds, but such a procedure is beset with pitfalls. A good example of the difficulties involved is the calculation of the deformation frequencies of the acetylene molecule. If a simple potential is assumed, involving only such characteristic angle constants, one obtains a false assignment of the fundamental frequencies which can only be avoided by taking into account a term in the potential energy which couples the two angle oscillators. The importance of such coupling terms has often been overlooked in calculations of the low-frequency fundamentals of molecules.

The calculation of the fundamental frequencies of even relatively simple molecules is a lengthy and tedious business, which can, however, be greatly shortened by making proper use of the symmetry of the equilibrium configuration of the molecule. There is further a general theorem due to Teller and to Redlich, which enables one to obtain useful results about the frequencies of isotopic molecules without making detailed calculations. This theorem is based on the fact that the vibrational or reduced mass of a normal mode can be expressed in the form of the product of the individual atomic masses divided by the total mass of the molecule and its moments of iner tia, these latter entering only if the vibration has the same symmetry respectively as a translation or infinitesimal rotation of the molecule. Since an isotopic substitution of hydrogen by deuterium does not change the potential, the effect on the frequencies is determined solely by the change in the reduced mass. Thus from the form of the vibrational mass the ratio of the products of frequencies of isotopic molecules can be deduced. The agreement is never perfect, owing to the fact that the observed frequencies require correction for the anharmonicity of the vibrations, but this general product theorem has been of great value in checking the assignments of frequencies without making lengthy calculations.

The rotational analysis is a much more involved problem than the vibrational analysis. This is due not only to the fact that a definite spectrum of rotational frcquencies is an idea quite foreign to classical theory and can be explained only on the basis of quantum kinematics, but also to the classical effect that internal vibrations in a rotating mechanical system give rise to Coriolis or vibrational-gyroscopic forces which greatly modify the rotational motion. The Coriolis forces as well as the simple centrifugal forces depend upon the nature of the vibrational potential, so that we obtain perturbations of the rotational structure of the absorption bands which depend upon the characteristic vibrational potential of the molecule. Thus whilst the simple theory of rigid rotating molecules leads to a classification of rotational spectra into those of spherical, symmetrical and asymmetrical top molecules, according to the nature of the ellipsoid of inertia, the exact theory requires a separate treatment for each individual molecule. A glance at recent work of this nature suffices to show that the quantum dynamical considerations involved are as thorough as any that have been necessary in the interpretation of the finer details of atomic theory. Whilst this complication might on one hand be regretted, it is on the other hand very satisfactory that the technical progress of experimental infra-red investigation has been so rapid that this branch of science will soon take equal rank with the sister science of atomic spectroscopy.

Although the advance in recent years has been so rapid, there is much more remaining to be done. The paucity of energy for wave-lengths greater than about $10 \mu$ in the spectra of heated bodies has been a factor which from the very beginning has retarded development. The technique, like any other concerned with the measurement of small quantities, is a difficult one, so that even to-day, in spite of the great potential importance of measurements in the infra-red both to pure science and to industry, the number of schools engaged in breaking new ground is very small. To this situation must be added the neglect of the commercial instrument maker which, though natural enough in the circumstances, means that every worker who wishes to be abreast of developments must be something of a precision instrument maker as well. Any improvement in design or technique which would enable measurements to be made with the same speed, reliability and ease with which they are made with photographic spectrographs would be an advance of the first magnitude.

An attack in this direction which promises well is the evaporograph, in which the spectrum is received on the blackened face of an extremely thin celluloid film, the reverse face of which is covered by a layer of oil molecules in equilibrium with the vapour phase. The minute differences of temperature caused by the energy variations in the spectrum produce changes in the thickness of the oil layer, which show up when its interference pattern is photographed with blue or ultra-violet light. 
In a review as short as this, applications can be only briefly indicated. For industry, important advantages are, first, that a sample may be examined without destroying or indeed interfering with it, and that very small quantities are required, and secondly, that since the spectrum between, let us say, $1 \mu$ and 30 is a unique property of any given substance, that spectrum may irf principle be used to identify the substance in any mixture in which it remains unaltered. In favourable cases concentrations as low as 1 in 10,000 may be recognized. If the substance is modified in the mixture, by association or otherwise, then its spectrum reflects the change and may be used as a source of information about it. So far as pure science is concerned, the greatest application of infra-red research in the near future will probably be made in conjunction with isotope chemistry, at first with deutero-substituted compounds, but later with other isotopes, carbon, nitrogen and oxygen in particular, as fast as they are made available in quantity. With this technique a knowledge of reaction mechanisms will be obtained which would scarcely be possible by any other method.

\section{THE FENLAND*}

\section{By Prof. J. Stanley Gardiner, F.R.S.}

$\mathrm{T}$ HE Fenland is an area of 73 miles by 36 miles, covering 1306 square miles, and comprises parts of the counties of Lincoln, Norfolk, Suffolk, Cambridge, Huntingdon and Northampton. It consists of Jurassic clays underlying glacial boulder clay, cut into basins and water courses by tidal and river actions. Some Jurassic islands were left, such as Ely, while peat formed in the swamps to the south, merging into a broad belt of silt against the sea. The area was not stable, and peat and silt in places alternate ; and often horizons of submerged forests are followed by swamp conditions. In the earliest period of history the Fenland was a flat expanse with scattered islands and ridges from the surrounding uplands, all these cultivated, while the swamps yielded rough produce for stock, the streams abundant fish, especially eels, and the marshes wild-fowl. Of course it must have been a paradise to the Romans by providing an outlet for their energies, the results of which are problematical. It is only certain that there was wide cultivation succeeded by decay. This was possibly due to the neglect of drainage works, complicated by a small change in land-level in progress towards the termination of the Roman occupation.

In the Norman period the condition of the Fenland may be inferred from the map of the Domesday settlements. It must have been affected by the relatively recent monastic foundations of Crowland, Ely, Ramsay, Thorney and Chatteris. For his recently issued studies on the Fenland, Mr. Darby has consulted all authorities, but they have helped little as to the economy in this AngloDanish period. His inferences are based on the Domesday settlement-peat lands do not allow

- The Medieval Fenland. By H. C. Darby. (Cambridge Studies In Economic History). Pp. xvii $+200+11$ plates. (Cambridge : At the University Press, 1940). 128. 6d. net.

The Draining of the Fens. By $\mathbf{H}$. C. Darby. (Cambridge Studies in Economic History). Pp. xix $+312+31$ plates. (Cambridge: At the University Press, 1940). 218, net. of the building of house foundations-the sizes of parishes and the distributions of fisheries, salt pans, meadow and other lands. The Wisbech estuary now became silted up and inundations followed. At some uncertain date the Eastern and Western Ouses were diverted, partly by artificial channels, to reach the sea at Lynn. Traffic to the surrounding lands apparently thronged the waterways. In the fourteenth century the Fenland was many times more prosperous than the surrounding upland. Strategically, it might be a centre of rebellion or of refugea comparison with part of southern Finland to-day is not inappropriate-but disturbances were largely dictated by neighbouring barons. Socially, there was no isolation and its people were not "endowed with any qualities of desperate wildness extracted by some mystical influence from reeds and rushes". Mr. Darby's economic study of this time is excellent, but he could have made it plainer to his readers had he presented a picture of the Fenland as it really was, its meandering rivers, open pools, marshes, sedge and litter lands, thickets and woods. Its peat is not the well-known product of moss but of the decay of sedge and litter; it is very alkaline.

The account of Tudor times in the Fens reveals a woeful condition of dire poverty. This was partially due to the upheaval that succeeded the dissolution of the monasteries, but every 'Court' was quite ineffective in maintaining the Fenland channels and drains. Parliament became interested in the matter and in 1534 passed the first Act for the preservation of birds ; wild-fowl were given a close season in June, July and August, and the taking of their eggs was prohibited "upon pain of imprisonment for one year". Later, there were great floods and many permanent 'drownings', and in $\mathbf{1 5 7 0}$ the sea broke in near Wisbech. Drainage 